enlarged $(>1.0 \mathrm{~cm})$ pelvic nodes $(\mathrm{P}=0.004),>3$ pelvic nodes $(\mathrm{P}<0.001)$, or involved common iliac $(\mathrm{P}<0.001)$, external iliac $(\mathrm{P}<0.001)$, internal iliac $(\mathrm{P}<0.001)$, or obturator $(\mathrm{P}=0.019)$ nodes, or prominent or enlarged paraaortic nodes at the time of CT-simulation $(\mathrm{P}<0.001)$. After a median follow-up of 11.3 months, there was no significant difference observed in terms of pelvic recurrence-free survival $(77.4 \%$ vs $80.4 \% ; \mathrm{P}=1.000)$, paraaortic recurrence-free survival $(936 \%$ vs $89.1 \% ; \mathrm{P}=0.95)$, distant metastasis-free survival $(77.4 \%$ vs $80.4 \% ; \mathrm{P}=0.780)$ and disease-free survival $(61.3 \%$ vs $69.6 \%$; $\mathrm{P}=0.472)$ between patients receiving EFRT versus Pelvic EBRT. The presence of enlarged $(>1.0 \mathrm{~cm}$ ) paraaortic lymph nodes during CT-simulation was independently associated with inferior disease-free survival (OR 8.45 [1.48 to 48.26]; $\mathrm{P}=0.016)$.

Conclusions Comparable survival outcomes were observed between cervical cancer patients receiving EFRT and Pelvic EBRT. Patients presenting with enlarged paraaortic nodes were found to have inferior disease-free survival despite having received EFRT.

\section{EPV088/\#577 OVERALL SURVIVAL AND TIME TRENDS IN CERVICAL CANCER IN ALMATY, KAZAKHSTAN}

${ }^{1} \mathrm{R}$ Bolatbekova*, ${ }^{2} \mathrm{D}$ Kaidarova, ${ }^{1} \mathrm{~N}$ Izbagambetov, ${ }^{1} \mathrm{~T}$ Valiyeva, ${ }^{3} \mathrm{Y}$ Kukubassov, ${ }^{1} \mathrm{~A}$ Aidarov, ${ }^{1} \mathrm{G}$ Bagatova, ${ }^{1} \mathrm{~B}$ Kudaibergenova, ${ }^{3} \mathrm{~A}$ Satanova, ${ }^{1} \mathrm{~A}$ Sarmenova. ${ }^{1}$ Almaty Oncology Center, Oncogynecology, Almaty, Kazakhstan; ${ }^{2}$ Kazakh Institute of Oncology and Radiology, Head of Kazior, Almaty, Kazakhstan; ${ }^{3}$ Kazakh Institute of Oncology and Radiology, Oncogynecology, Almaty, Kazakhstan

\subsection{6/ijgc-2021-IGCS.156}

Objectives In Kazakhstan standardized incidence of CC was 17.2 per 100,000 , the mortality rate was 6 per 100,000 for 2020. The overall survival (OS) of CC in Kazakhstan was $52,5 \%$ (95\%CI:50,7-54,2). The CC Screening program uses cytology (Pap-smear) from 2008 for women 30-70 years every 4 years. Almaty remains the country's largest city with high cancer incidence and mortality. The purpose was to analyze time-trends for 2005-2020 and OS from CC in Almaty.

Methods Incidence and mortality were sourced from National Cancer Registry database. All rates were directly age-standardized. Data on survival were obtained from reports. OS was performed using the Kaplan-Meier method. The statistical analysis was performed with SPSS23.0.

Results The total number of registered women with CC in Almaty was in 2462. CC incidence is decreased from 16 to 13.4 per 100000 female population for last 15 years, Mortality $\mathrm{i}$ from 5.8 to 4.6 per 100000 female population. The average age of women with CC in 2016 was $50.8 \pm 11.7$. 241 cases included: most of them at 1st stage-128(50.3\%) 90 $(35.3 \%)$ in stage II, $18(7 \%)$ in stage III, 5(1,9\%) in an advanced stage. 38 women were dead from CC. The OS was $81,7 \pm 0.88 \%$ (95\%CI: 80,82-82,58)

Conclusions The CC incidence and mortality is lower in comparison with the republican values associated with better screening service and control in Almaty. The OS from CC in Almaty was higher than Kazakhstan regional average. Despite of positive results of CC screening, mortality rate is high compared to developed countries, which makes it necessary to introduce HPV-screening and HPV-vaccination

\section{EPV089/\#619 OUTCOMES AFTER ESTABLISHMENT OF A PILOT CERVICAL CANCER NAVIGATION PROGRAM AT A TERTIARY TANZANIAN ACADEMIC HOSPITAL}

${ }^{1} \mathrm{~A}$ Hari ${ }^{*},{ }^{2} \mathrm{~L}$ Maillie, ${ }^{3} \mathrm{~K}$ Schroeder, ${ }^{4} \mathrm{~A}$ Yussuph. ${ }^{1}$ UC Irvine, Gynecologic Oncology, Orange, USA; ${ }^{2}$ cahn Mount Sinai, School of Medicine, New York City, USA; ${ }^{3}$ Duke University, Pediatric Oncology, Durham, North Carolina, USA; ${ }^{4}$ Bugando Medical Center, Obstetrics and Gynecology, Mwanza, Tanzania, Tanzania

\subsection{6/ijgc-2021-IGCS. 157}

Objectives Standard of care for advanced locoregional cervical cancer is primary chemoradiation (pCRT). The majority of patients prescribed pCRT for advanced cervical cancer do not complete their treatment in Northern Tanzania secondary to a complex web of health and socioeconomic stressors. Bugando Medical Center (BMC), a tertiary academic care center in Mwanza, Tanzania, established a cervical cancer navigation program (CCNP) to overcome these barriers.

Methods Funding was provided by the International Mennonite Foundation. CCNP consisted of a navigator, hostel manager, and project manager. Patients were provided food, transportation, housing, labs, imaging, and treatment costs as needed. Patients were also given counseling, education, and social support throughout the course of pCRT.

Results 71 consecutive patients referred to BMC with newly diagnosed cervical cancer were enrolled between January 2020 and December 2020. These patients were not surgical candidates and were prescribed pCRT. Their age range was 30 to 89 years (median 50) and the majority of patients had squamous cell $(70,99 \%)$ and 1 patient $(1 \%)$ had adenocarcinoma. $26(37 \%)$ were HIV positive or unknown and 45 (63\%) were HIV negative. During the year, 53 (75\%) patients were able to fully complete recommended pCRT and 18 (25\%) are still undergoing treatment; no one was lost to follow up during treatment.

Conclusions Social determinants of health play a role in timely completion of pCRT so in order to address these, a pilot CCNP was successfully implemented at BMC and supported 71 patients financially, medically, and psychosocially through their pCRT.

\section{EPV090/\#629 PATTERNS OF CARE AND OUTCOMES OF ADENOCARCINOMA OF CERVICAL CANCER POST TREATMENT - RETROSPECTIVE STUDY FROM A TERTIARY CARE CENTRE IN SOUTH INDIA}

${ }^{1} \mathrm{JK}$ Ramireddy*, ${ }^{1} \mathrm{~N}$ Oliver John, ${ }^{1} \mathrm{~A}$ Sathyamurthy, ${ }^{1} \mathrm{~J}$ Priyadarshini Rao, 'S Milton, ${ }^{1} \mathrm{~V}$ Thomas, ${ }^{2} \mathrm{~A}$ Thomas, ${ }^{1} \mathrm{~T}$ Ram. ${ }^{1}$ Christian Medical College and Hospital, Vellore, Department of Radiation Oncology, Vellore, India; ${ }^{2}$ Christian Medical College, Vellore, Gynecologic Oncology, Vellore, India

\subsection{6/ijgc-2021-IGCS. 158}

Objectives Non - squamous histologies such as adenocarcinoma of the cervix might have an aggressive clinical course. There is sparse literature on tailoring treatment in adenocarcinoma cervix. In this study we plan to do a retrospective review of patients with this entity.

Methods The medical records of 2462 patients with cervical cancer between January 2008 to December 2018 were collected. The records of 180 patients who had histologically proven adenocarcinoma cervix were reviewed. The 